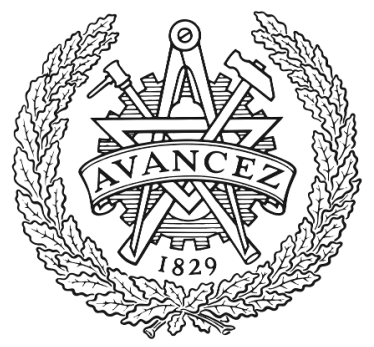

CHALMERS

UNIVERSITY OF TECHNOLOGY

\title{
An Adapted Evasive Manoeuvre Assist Function for Over-Reactive and Under-Reactive Drivers
}

Downloaded from: https://research.chalmers.se, 2023-04-26 10:24 UTC

Citation for the original published paper (version of record):

Harinath, P., Kittane, S., Yang, D. et al (2020). An Adapted Evasive Manoeuvre Assist Function for Over-Reactive and Under-Reactive Drivers. Lecture Notes in Mechanical Engineering: 1017-1026. http://dx.doi.org/10.1007/978-3-030-38077-9_118

N.B. When citing this work, cite the original published paper. 


\title{
An Adapted Evasive Manoeuvre Assist Function for Over-Reactive and Under-Reactive Drivers
}

\author{
Preetham Harinath ${ }^{1,2}$, Santusht Vasuki Kittane ${ }^{1,2}$, Derong Yang ${ }^{1}$, Lars \\ Drugge $^{2}$, Mats Jonasson ${ }^{3}$ \\ ${ }^{1}$ Department of Active Safety and Vehicle Dynamics, Volvo Car Group, \\ Gothenburg, SE-405 31, SWEDEN \\ ${ }^{2}$ Department of Aeronautical and Vehicle Engineering, Vehicle Dynamics, The Royal \\ Institute of Technology (KTH), Stockholm, SE-100 44, SWEDEN \\ 3 Department of Mechanics and Maritime Sciences, Chalmers University of \\ Technology, SE-412 96 Gothenburg, SWEDEN
}

\begin{abstract}
In the present paper, an Evasive Manoeuvre Assist (EMA) function is designed to adapt to different types of drivers, by an optimised steering torque overlay. The existing EMA function amplifies the driver steering inputs using a feed-forward controller which might not necessarily help an over-reactive driver. There exists a need for an EMA which adapts to different drivers as it minimises the risk of collision and gives the driver an experience of good control. The focus of this paper is to identify and define a proper steering sequence reference model for closed-loop feedback control design. A simple single-point preview model is designed first to calculate the reference steering angle. A few test scenarios are set-up using the IPG CarMaker ${ }^{\mathrm{TM}}$ simulation tool. The reference model is then calibrated with respect to the amplitude and frequency of the steering sequence by offline optimisation to obtain the optimal steering profile. A feedback controller is then designed using this reference model. The robustness of the function is verified in real-time, using a Volvo rapid-prototype test vehicle.
\end{abstract}

Keywords: collision avoidance - Evasive Manoeuvre Assist (EMA) . over-reactive drivers · under-reactive drivers · safe zone concept.

\section{Introduction}

Earlier studies have shown that most drivers do not steer enough when faced with the risk of rear-end collisions even though about $50 \%$ of the critical situations could have been avoided had they steered enough [1]. Field tests have also shown that more than $50 \%$ of the people have chosen to steer at imminent side collisions, e.g. with oncoming vehicles at intersections and that drivers are more likely to brake even when the optimal approach would be to steer alone, or to steer in combination with braking [2], [3]. The Evasive Manoeuvre Assist (EMA) function is emerging on the market as an active safety function, which assists the driver to avoid imminent frontal collisions. 
The existing EMA function [8] always amplifies the driver steering inputs via a short constant pulse of Electric Power Assisted Steering (EPAS) torque overlay [4-6]. Two cases highlight the problem tackled in this paper, considering the assistance torque is constant and independent of the driver's reaction.

- Driver under-reacts: With a constant assistance torque being provided in this case, the under-reactive driver might still cause a collision because the car does not have the necessary lateral deviation owing to insufficient assistance torque.

- Driver over-reacts: The driver's over-reaction might deviate the car excessively, resulting in the car going off-road, colliding head-on with another car/obstacle or trigger instability. Combining the driver's reaction with a constant assistance torque would just make the car oversteer and eventually lose stability.

Contrary to a constant assistance torque in the existing EMA function [7], a continuously varying torque interference during an evasive manoeuvre which accommodates different drivers' reaction is proposed in the present paper. The proposed function involves the generation of a reference steering angle sequence and a controller to provide a steering torque overlay.

\section{Reference Modelling}

The reference model was created by calculating the curvature needed to negotiate the manoeuvre and this curvature was translated to the steering wheel angle using the bicycle model. The reference model is similar to the driver model in that the lateral deviation is set based on a minimum clearance threshold between the ego vehicle and the obstacle akin to an S-shaped escape path shown in Fig. 1.

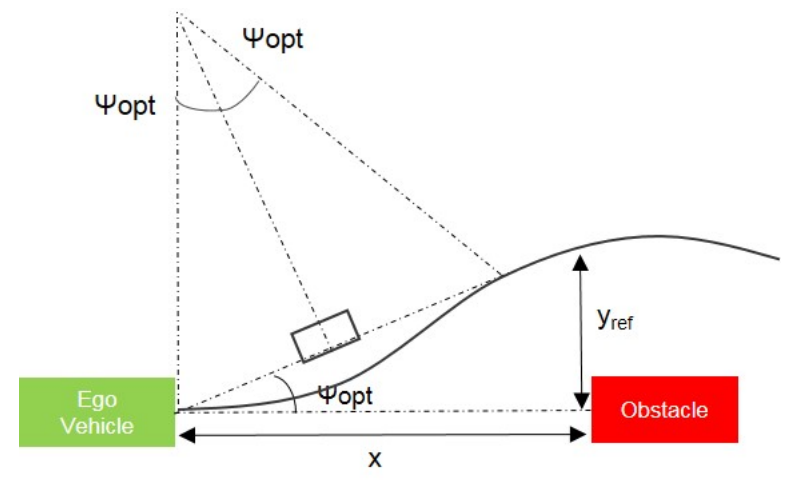

Fig. 1: Reference preview definition 
The angle between the vehicle direction and the horizontal $\left(\psi_{\text {opt }}\right)$ can be approximately translated to the curvature $(\mathrm{C})$ needed to reach the point ( $\mathrm{x}$, $y_{\text {ref }}$ ) using eq(1).

$$
C=\frac{2 \psi_{\text {opt }}}{\sqrt{x^{2}+y_{\text {ref }}^{2}}}
$$

With the calculated curvature as the amplitude, the curvature signal was generated in the form of a sine wave. The curvature signal was transformed to the steering angle $(\delta)$ sequence using a simple kinematic equation of a bicycle model of a vehicle with wheelbase (L) and understeer gradient $\left(K_{u s}\right)$ as shown in eq(2).

$$
\delta=L * C+K_{u s} * V_{x}^{2} * C
$$

Thus, the reference steering sequence generated takes the vehicle parameters into consideration and also adapts suitably when the velocity changes.

\section{Safe Zone Control}

During an evasive manoeuvre, a safe reaction of the driver might range from a steering input which narrowly clears the obstacle in front to an input which easily clears the obstacle with certain margins, but just stays within the road boundary. The traversed paths corresponding to the two extreme safe reactions of the drivers thus form a region [9], termed as the safe zone as depicted in Fig. 2. Rather than generating just one reference for EMA control, this concept uses two

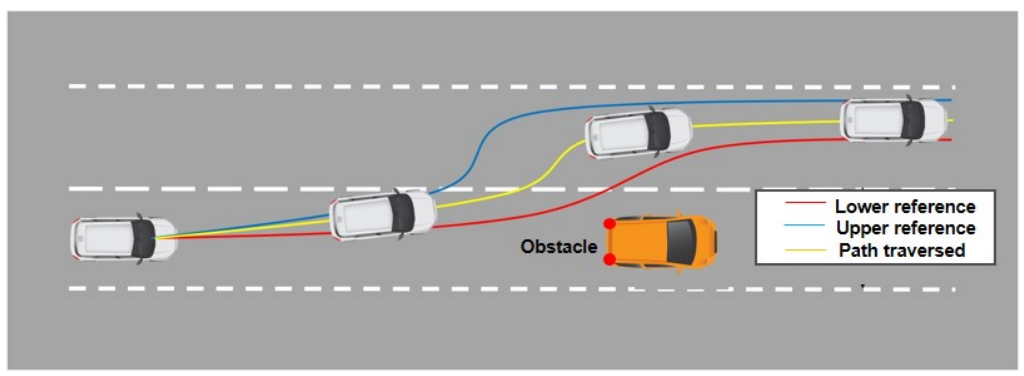

Fig. 2: Illustration of the EMA Safe Zone Control strategy

references to accommodate a multitude of driver reactions. The lower reference, aiming to achieve the minimum required lateral deviation will correspond to a less aggressive manoeuvre than the upper reference, which instead aims to restrict the car away from the road boundary. 


\subsection{Control Strategy}

To analyse the amplitude and duration of the two references in the safe zone control strategy, batch simulations were conducted using IPG CarMaker ${ }^{\mathrm{TM}}$ on a validated car model. The control architecture employed for these batch simulations has been indicated in Fig. 3.

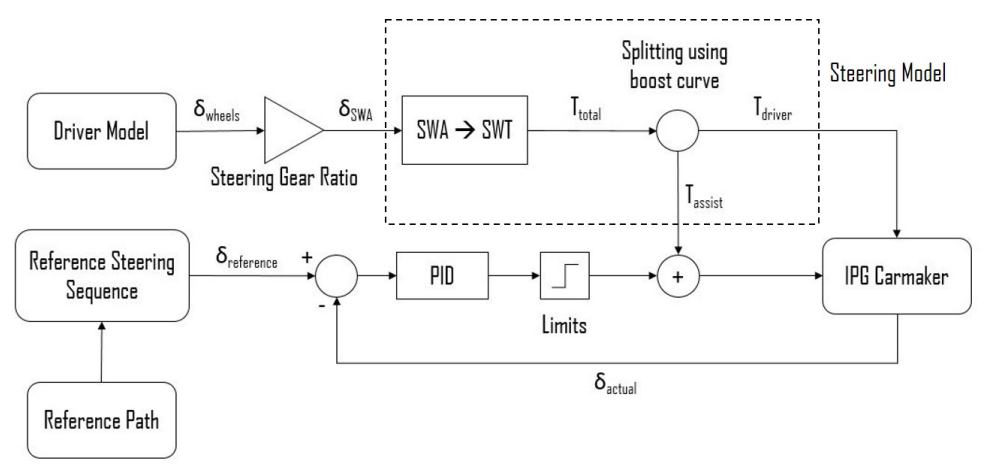

Fig. 3: Control architecture

By varying the point the driver aims at, different paths and hence different steering sequences can be generated as shown in Fig. 4.

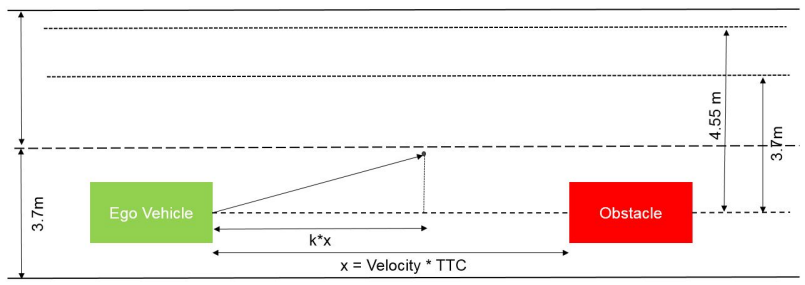

Fig. 4: Path generation (TTC: Time To Collision)

The variation was done as a function of the initial distance $(\mathrm{x})$ when the driver initiates the manoeuvre using the factor $\mathrm{k}$. Further, the steering sequence duration was also varied as a function of $(2 *$ TTC). Data such as lateral deviation, time interval, lateral acceleration and steering wheel angle was collected for each manoeuvre. The results of the simulations were analysed with cost functions representing the objectives of each of the references.

The cost function for the lower reference intends to find the case having a lateral deviation (y) closest to (but also higher than) the minimum lateral deviation 
$\left(y_{\min }\right)$ and the longest time $\left(t_{l}\right)$ to achieve it. The cost function for the lower reference with weighting factors $\left(w_{1} \& w_{2}\right)$ has been indicated in eq(3).

$$
\operatorname{minimize}\left(w_{1} *\left(y-y_{\min }\right)-w_{2} * t_{l}\right)
$$

The cost function for the upper reference intends to find the case having a final lateral deviation $\left(y_{f}\right)$ closest to (but lesser than) the maximum possible lateral deviation $\left(y_{\max }\right)$ and the shortest time $\left(t_{s}\right)$ to achieve it. The cost function with weighting factors $\left(w_{3} \& w_{4}\right)$ for the upper reference has been indicated in eq(4).

$$
\operatorname{minimize}\left(w_{3} *\left(y_{\max }-y_{f}\right)+w_{4} * t_{s}\right)
$$

The weighting factors in the cost functions indicated in eq(3) \& eq(4) used the normalised sub-cost functions to reflect the relative importance of a particular case in the matrix set of data obtained from conducting batch simulations.

The outcome of these batch simulations together with the cost functions was two factors concerned with defining the steering sequence. One of the factors defines the longitudinal position of the point the driver aims at, as a function of the initial distance and the other factor defines the duration of the manoeuvre as a function of $(2 *$ TTC). The set of these two parameters best fitting the objectives of the upper and lower references were selected for that particular manoeuvre according to the cost functions. These parameters served as initial values when real-time testing was performed.

\subsection{Reference Update}

The upper and lower references vary in both amplitude and duration. As shown in Fig. 5, it can be observed that there are some manoeuvres which exceed the region covered by the two references, namely grey areas (indicated by the red circles).

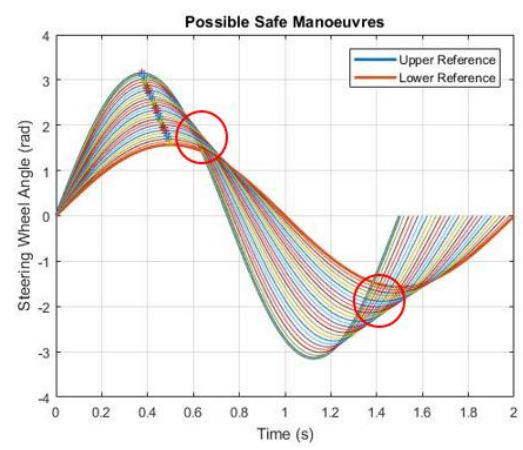

Fig. 5: Possible safe manoeuvres

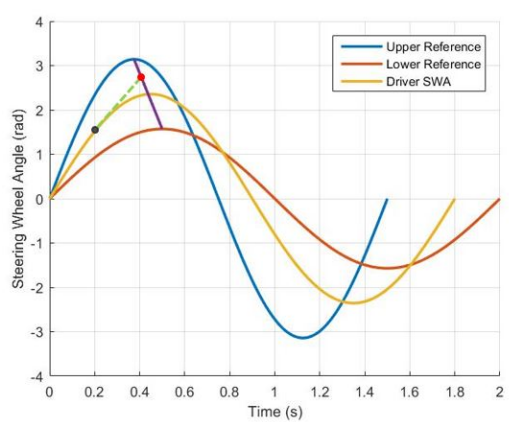

Fig. 6: Upper reference update

To account for these grey areas, there was a requirement to update the reference suitably. Since an evasive manoeuvre lasts only for a few seconds, it is 
critical that the updating duration be set accordingly. Depending on the Time To Collision (TTC), the updating duration has to be set and updating has to be stopped. Updating the reference is stopped after a certain predefined duration because while the purpose of the reference is to provide intuitive control to the driver, it is also to not play along to the driver's faulty reactions. Thus, the upper and lower reference is fixed after a certain duration (called the update time) for the rest of the manoeuvre and suitable control action is applied.

In Fig. 5, it was observed that the positive peaks of all possible manoeuvres lie on the same line. For the upper reference, updating was done by extrapolating the driver steering sequence at a sample time corresponding to the update time to intersect the line joining the amplitudes of the original upper and lower reference, as shown in Fig. 6 . The point of intersection (indicated by the red dot in Fig. 6) would give the amplitude of the updated upper reference. The time corresponding to the new amplitude is the quarter of the duration of the entire updated sequence.

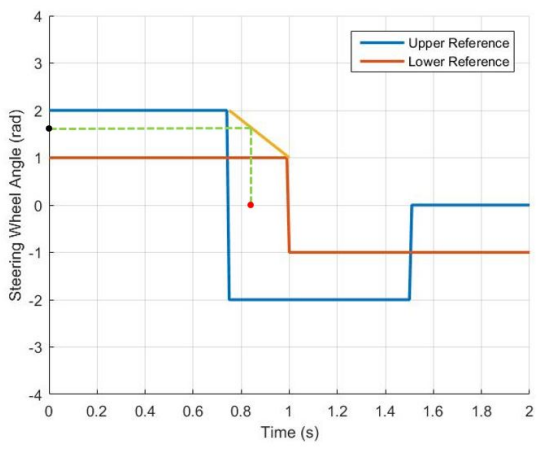

Fig. 7: Lower reference update

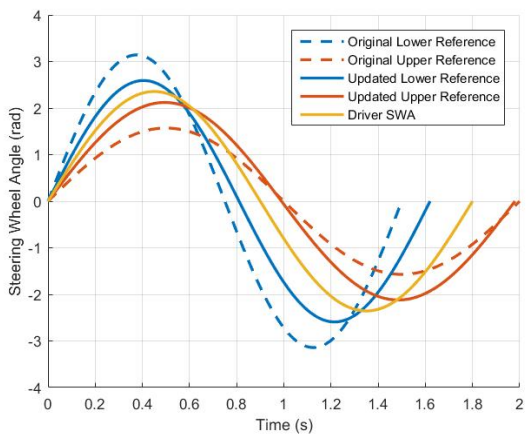

Fig. 8: Original and updated references

The updating of the lower reference also happens very similar to the upper reference. The amplitude of the updated lower reference is chosen to be that of the driver's at the sample time corresponding to the update time. If the driver has started stabilising the car in the evasive manoeuvre (steering wheel angle rate $<0)$ at the update time, then the driver's highest achieved steering wheel angle before the steering wheel angle rate changes sign is taken as the amplitude. The square wave substitutes of the two references are used to find the half duration of the updated lower reference, as shown in Fig. 7.

The original and updated references along with the driver applied steering sequence is shown in Fig. 8. The updated references had the advantage of accounting for the grey areas effectively and being more compatible to the driver's reaction thus providing intuitive control. 


\section{Countermeasures}

One of the main challenges of this function was to adapt it to a variety of drivers. For the function to work in conjunction with different driver behaviours satisfactorily, it was necessary to classify them in a simple yet effective way to encompass the wide spectrum of driver reactions. For this purpose, different types of driver reactions were broadly classified into three types: moderately safe, excessive under-reaction and excessive over-reaction. Each type of driver reaction was further sub-classified based on the driver's initial aggressiveness. An aggressive sub-classification would correspond to the driver stabilising the car before the update time and a passive one would correspond to stabilising after the update time. The countermeasures were tuned with two objectives in mind: to help the driver to the maximum extent and to provide adapted and intuitive control.

Table 1 shows the countermeasures associated with different unsafe driver reactions.

Table 1: Countermeasures

\begin{tabular}{|c|c|c|}
\hline Driver reaction & Type & Countermeasures \\
\hline Moderately safe & Aggressive & Control with the updated references with scaling factors on the duration \\
\hline$($ Update time $=$ Upper reference duration $/ 4)$ & Passive & (1.1* updated lower reference duration and $0.9 *$ updated upper reference duration) \\
\hline \multirow{2}{*}{$\begin{array}{c}\text { Excessive under-reaction } \\
\text { (Update time }=\text { Lower reference duration } / 4 \text { ) }\end{array}$} & Aggressive & Control with only the original lower reference \\
\hline & Passive & $\begin{array}{l}\text { Control with only the updated lower reference, } \\
\text { but with a longer duration than the original lower reference }\end{array}$ \\
\hline \multirow{2}{*}{$\begin{array}{c}\text { Excessive over-reaction } \\
(\text { Update time }=\text { Upper reference duration } / 4)\end{array}$} & Aggressive & $\begin{array}{l}\text { Control with only the updated upper reference, } \\
\text { but with a shorter duration than the original upper reference }\end{array}$ \\
\hline & Passive & Control with only the original upper reference \\
\hline
\end{tabular}

For a moderately safe driver, there is no control intervention if the driver input lies in the common region enclosed by the updated references. The closest reference is used for control if this region is surpassed. For the excessive underreaction and excessive over-reaction cases, only one reference is used for control. The control action is delivered using a PID controller. The scaling factors mentioned in Table 1 were obtained upon extensive real-time testing based on several drivers' subjective feedback.

\section{Results}

Upon generating the references and designing the updating algorithm, the next step was to test the function in real-time vehicle testing using the baseline values obtained from batch simulations. 


\subsection{Test Scenarios}

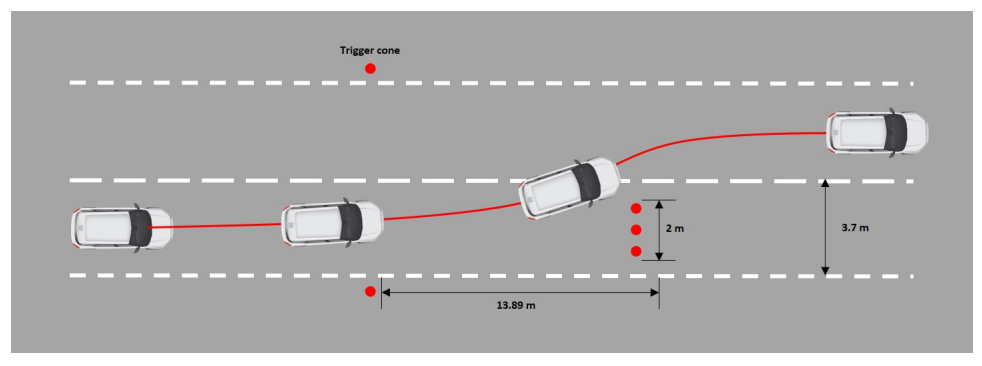

Fig. 9: Test setup for EMA function

The tests were carried out in a Volvo V40 Cross Country equipped with a MicroAutoBox dSPACE controller. The effectiveness of the countermeasures was evaluated using OxTS ${ }^{\mathrm{TM}}$ GPS-reference instrumentation [10], as well as subjectively by various drivers in the Volvo rapid prototype vehicle.

Fig. 9 illustrates the test scenario used. The distance between the driver's initial steering reaction and the obstacle was fixed to be at a distance of 13.89 $\mathrm{m}$, which corresponds to a TTC of $1 \mathrm{~s}$ if the vehicle travels at $50 \mathrm{~km} / \mathrm{h}$. The size of the obstacle was also defined to be $2 \mathrm{~m}$ wide. The speed of the manoeuvre was varied to obtain different TTCs.

Case 1: $50 \mathrm{~km} / \mathrm{h}$ over-reactive driver with 1s TTC 1s: Fig. 10 illustrates the performance of the controller for an over-reactive driver during an evasive manoeuvre at around $50 \mathrm{~km} / \mathrm{h}$.

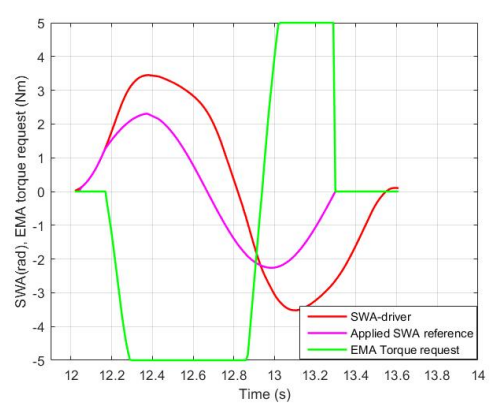

((a)) Updated SWA reference

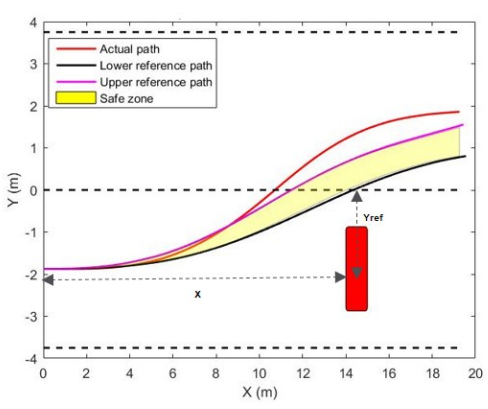

((b)) Global path

Fig. 10: Applied steering torque, Steering Wheel Angle (SWA) reference and global path traversed at $50 \mathrm{~km} / \mathrm{h}$ 
The over-reactive and under-reactive driver steering wheel angle references were derived from the paths as mentioned in Fig. 1. The profiles were then calibrated and used to predict the driver's reaction to generate the updated references as shown in Fig. 10(a). In accordance with the updated steering wheel angle reference, the controller provides a negative EMA torque request (represented by the green curve in Fig. 10(a)) to counteract the initial over-reaction of the driver and then further aids the driver in stabilising the car after evading the obstacle. Fig. 10(b) represents the path traversed by the vehicle during this evasive manoeuvre. The driver clearly goes beyond the bounds of the safe zone and the controller correspondingly provides a resistance torque to prevent further over-reaction.

Case 2: $90 \mathrm{~km} / \mathrm{h}$ under-reactive driver with TTC - 0.55s: Fig. 11 represents a scenario where the driver under-reacts and fails to provide the necessary steering torque to clearly evade the obstacle.

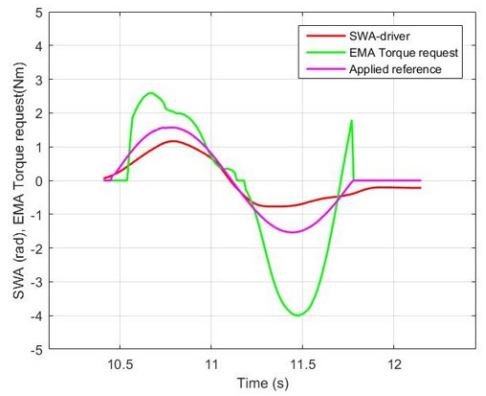

((a)) Updated SWA reference

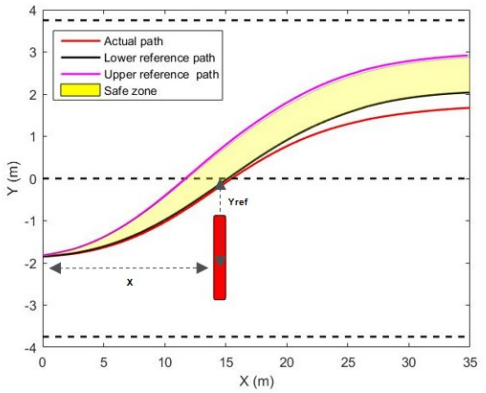

((b)) Global path

Fig. 11: Applied steering torque, Steering Wheel Angle (SWA) reference and global path traversed at $90 \mathrm{~km} / \mathrm{h}$

Fig. 11(b) illustrates the path traversed by the vehicle and also the lower and upper reference paths which represents the safe zone. The intended path of the vehicle is below the lower reference path which shows that the driver under-reacts. Fig. 11(a) depicts how an EMA torque request assists the driver, contrary to the previous case.

It is important to note that the torque output of the controller has been constrained (Fig. 3) in order to ensure a smooth operation of the steering actuator. As a result, the actual steering wheel angle rarely coincides with the reference sequence. 


\section{Conclusions}

The objective of this paper was to design a robust EMA function which could adapt to both under-reactive and over-reactive drivers. The safe zone concept was used for control instead of a single reference because it considers a myriad of driver reactions and makes the assistance more robust. Since the reference steering sequences vary in both amplitude and duration, updating the references, in accordance with the driver's behaviour, was necessary to provide highly intuitive control interventions. The original references and the update time at which the references are modified play a critical role in the performance of the system. Real-time vehicle testing showed that the designed EMA function can accommodate a defined set of driver reactions, in order to provide effective torque assistance/resistance intervention with acceptable robustness.

Future work is to further validate the controller performance by real-time testing with a driving robot, which can provide an objective analysis with and without the proposed function.

\section{References}

1. Hartmann, B., Eckert, A., Rieth, E. and Sevenich, M., Emergency Brake \& Steer Assist - The Integration of Emergency Brake and Steer Assistance Taking Driver Behaviour in Emergency Situation into Account, 20th Aachen Colloquium Automobile and Engine Technology, 11-011, Germany, 2011

2. Schittenhelm, H., Fahrverhalten und reaktionszeiten in kritischen situationen, VDIBericht 1911, 2005

3. Adams, L.D., 'Review of the literature on obstacle avoidance manoeuvres: braking versus steering', University of Michigan Transportation Research Institute, Ann Arbor, USA. Report No. UMTRI-94-19, 1994

4. Yang, D., Jonasson, M., Hallerd, T., Johansson, R., 'Evaluation of an evasive manoeuvre assistance system at imminent side collisions', AVEC16, 2016

5. Shah, J., Zegelaar, P. and Best, M., 'Performance of Emergency Steer Assist in Front Wheel and Rear Wheel Steering Vehicle', AVEC14, 2016

6. Breuer.J, 'Analysis of driver vehicle interactions in an evasive manoeuvre - results of moose test studies', Daimler-Benz AG, 98-S2-W-35, 1998

7. Lokur, P. and Wang, Y., 'Effectiveness of Evasive Manoeuver Assist Function for Different Driver Skills', Chalmers University of Technology, Master dissertation, 2016

8. Jonasson, M. and Gustafssson, T., Driver assist arrangement, Grant patent no. US9643604B2, 2017

9. Yang, D., Jonasson, M. and Silvlin,J., Method and system for providing a driver behaviour adapted evasive manoeuvre, Patent application no. US20160339910A1, 2016.

10. Online: https://www.oxts.com/, 'Oxford Technical Services' 